\title{
Calcolo ottimale della dose di L-tiroxina nel paziente obeso tiroidectomizzato
}

\author{
Bernadette Biondi ${ }^{1}$
}

Pubblicato online: 10 luglio 2020

(c) The Author(s) 2020

Commento a:

Evaluation of thyroid hormone replacement dosing in overweight and obese patients after a thyroidectomy. V. Papoian, D. Ylli, E.A. Felger, L. Wartofsky, J.E. Rosen.

Thyroid (2019) 29(11):1558-1562

Il National Institute of Health (NIH) ha recentemente stimato che circa il $23 \%$ della popolazione negli Stati Uniti è in sovrappeso, il $37 \%$ è obesa e il 7,7\% è estremamente obesa [1].

Il numero di pazienti tiroidectomizzati è aumentato nel corso degli anni [2] e il rischio di un "over and under treatment" durante la successiva terapia sostitutiva con LTiroxina (LT4) è considerato particolarmente frequente nel paziente obeso sottoposto a tiroidectomia totale.

La dose iniziale di LT4 viene infatti calcolata sulla base del peso corporeo del paziente ed è generalmente di circa $1,6 \mu \mathrm{g} / \mathrm{Kg}$ nel soggetto tiroidectomizzato [3]. Tale dose viene successivamente modificata nel corso del tempo di osservazione fino a ottenere l'eutiroidismo e, quindi, la normalizzazione dei valori del TSH e degli ormoni tiroidei. L'insieme dei dati della letteratura ha infatti evidenziato che, oltre al peso e alla composizione corporea, l'età, il sesso e alcune condizioni fisiologiche (come la gravidanza) o patologiche (tra cui, soprattutto, patologie cardiovascolari e renali) sembrano essere i parametri più importanti da valutare per il raggiungimento di una terapia sostitutiva corretta e personalizzata del paziente con ipotiroidismo post-chirurgico [3].

$\triangle$ B. Biondi

bebiondi@unina.it

1 Dipartimento di Medicina Clinica e Chirurgia, Università Federico II di Napoli, Napoli, Italia
Dati della letteratura suggeriscono che l'obeso con ipotiroidismo post-chirurgico riceve frequentemente un overtreatment perché trattato con dosi particolarmente elevate di LT4 in considerazione dell'elevato peso corporeo che, generalmente, non viene corretto per l'indice di massa corporea.

Si rende, pertanto, necessario un metodo utile per calcolare la dose corretta di LT4 nella popolazione obesa onde evitare gli elevati costi sanitari per il più frequente monitoraggio della terapia sostitutiva, rispetto alla popolazione normopeso, e il più elevato rischio cardiovascolare associato alla possibilità di ipertiroidismo o ipotiroidismo iatrogeni.

Alcuni studi hanno proposto differenti strategie di calcolo della dose di LT4 nel paziente obeso oltre alla valutazione del peso corporeo, tra cui il calcolo basato sulla massa magra, il peso corporeo ideale o la valutazione della superficie corporea $[4,5]$.

Particolarmente interessanti sono i risultati di questo studio retrospettivo su 428 pazienti sottoposti consecutivamente a tiroidectomia totale presso la Georgetown University/Washington Hospital Center di Washington tra il 2012 and 2015 [6]. Gli autori hanno utilizzato l'indice di massa corporea (BMI) come parametro utile per ottenere un calcolo semplice della dose di LT4 nel paziente obeso. Un totale di 255 pazienti, consecutivamente sottoposti a tiroidectomia totale per patologia benigna, è stato valutato a tale scopo; di questi, solo 114 casi sono stati analizzati per la disponibilità dei dati di follow-up prima di raggiungere l'eutiroidismo durante LT4. L'età media dei pazienti era di 55 anni e l' $84 \%$ erano donne. Il $48 \%$ dei pazienti erano in sovrappeso o francamente obesi (il $23 \%$ aveva un BMI $<25$, il $29 \%$ tra 25 e 29 , il $20 \%$ tra 30 e 34 , il $17 \%$ tra 35 e 39 e l' $1 \%>40$ ). Il $52 \%$ dei pazienti era stato sottoposto a tiroidectomia totale per gozzo sintomatico, il $45 \%$ per noduli tiroidei sospetti di malignità e il $3 \%$ per morbo di Basedow. 
La relazione tra dose di LT4 e il peso corporeo, calcolata secondo modelli di regressione lineare, differiva in rapporto alla categoria di BMI dei pazienti. La dose di LT4 era rispettivamente di:

- $1,76 \mu \mathrm{g} / \mathrm{kg}$ per i pazienti con BMI <25;

- 1,47 per quelli con BMI 25-29;

- 1,42 per i pazienti con BMI 30-34;

- 1,27 per quelli con BMI 35-39;

- 1,28 per BMI $>40$.

Tali dati supportano l'importanza di considerare il BMI, oltre al peso corporeo, per il calcolo della dose corretta di LT4 nel paziente obeso. Il BMI, infatti, è risultato un parametro semplice e corretto per la valutazione della dose sostitutiva iniziale di LT4 nell'obeso tiroidectomizzato.

Le conclusioni di questo studio confermano dati di precedenti studi retrospettivi [7] e dimostrano che i pazienti obesi richiedono una dose più bassa di LT4 durante terapia sostitutiva rispetto ai pazienti normopeso.

I limiti di questo studio sono legati alla sua natura retrospettiva, al numero relativamente limitato di pazienti e alla valutazione di dati estratti da un registro elettronico e, quindi, all'assenza di informazioni sulla corretta e/o costante assunzione della LT4 da parte dei pazienti e alla possibilità di interferenze farmacologiche o nell'assorbimento della LT4.

Studi prospettici su una casistica più ampia potranno meglio chiarire l'insieme dei parametri che devono essere considerati per una corretta terapia sostitutiva con LT4, soprattutto nel paziente obeso.

Ringraziamenti Open access funding provided by Università degli Studi di Napoli Federico II within the CRUI-CARE Agreement.

Nota della casa editrice Springer Nature rimane neutrale in riguardo alle rivendicazioni giurisdizionali nelle mappe pubblicate e nelle affiliazioni istituzionali.
Open Access This article is licensed under a Creative Commons Attribution 4.0 International License, which permits use, sharing, adaptation, distribution and reproduction in any medium or format, as long as you give appropriate credit to the original author(s) and the source, provide a link to the Creative Commons licence, and indicate if changes were made. The images or other third party material in this article are included in the article's Creative Commons licence, unless indicated otherwise in a credit line to the material. If material is not included in the article's Creative Commons licence and your intended use is not permitted by statutory regulation or exceeds the permitted use, you will need to obtain permission directly from the copyright holder. To view a copy of this licence, visit http://creativecommons.org/licenses/by/4.0/.

\section{Bibliografia}

1. Flegal K, Kruszon-Moran D, Carroll M et al (2016) Trends in obesity among adults in the United States, 2005 to 2014. J Am Med Assoc 315:2284-2291

2. Sun GH, DeMonner S, Davis MM (2013) Epidemiological and economic trends inpatient and outpatient thyroidectomy in the United States, 1996-2006. Thyroid 23:727-733

3. Biondi B, Wartofsky L (2014) Treatment with thyroid hormone. Endocr Rev 35:433-512

4. Mele C, Tagliaferri MA, Pagano L et al (2019) Levo-thyroxine replacement in obese adults: the role of metabolic variables and aging on thyroid testing abnormalities. J Clin Endocrinol Metab 104(12):6265-6274

5. Santini F, Marzullo P, Rotondi M et al (2014) Mechanisms in endocrinology: the crosstalk between thyroid gland and adipose tissue: signal integration in health and disease. Eur $\mathrm{J}$ Endocrinol 171(4):R137-R152

6. Papoian V, Ylli D, Felger EA et al (2019) Evaluation of thyroid hormone replacement dosing in overweight and obese patients after a thyroidectomy. Thyroid 29(11):1558-1562

7. Ojomo KA, Schneider DF, Reiher AE et al (2013) Using body mass index to predict optimal thyroid dosing after thyroidectomy. J Am Coll Surg 216:454-460 